\title{
Phytosociological Relevé Database of Japan (PRDB)
}

\author{
Nobuyuki Tanaka
}

\begin{abstract}
The Phytosociological Relevé Database of Japan (PRDB; GIVD ID AS-JP-001) is the database composed of many plot (relevé) data on every type of vegetation, which have been collected throughout Japan with the phytosociological method. A relevé datum comprises the ID no., survey date, surveyor name, references, surveyed area, location, environmental data, community structure, species name and their dominance, etc. The PRDB provides the information on the distribution of a variety of species. The PRDB has been constructed by research projects about the assessment of climate change impact on vegetation and biodiversity.
\end{abstract}

Keywords: every type of vegetation; vegetation plot; whole Japan.

\section{Phytosociological Relevé Database of Japan (PRDB)}

Scope: Phytosociological Relevé Database of Japan (PRDB) is the database composed of many plot (relevé) data on every type of vegetation, which have been collected throughout Japan with the phytosociological method. The PRDB provides the information on distribution of a variety of plant species. The PRDB has been constructed under research projects on impact assessment of climate change on vegetation and biodiversity. Status: emerging Period: $1950-2008$

Database manager(s): Nobuyuki Tanaka (ntanaka@affrc.go.jp)

Owner: Forestry and Forest Products Research Institute

Web address: http://www.ffpri.affrc.go.jp/labs/prdb/EnglishVer/index-e.html
Availability: according to a specific agreement
Online upload: no
Online search: no

Database format(s): FVD

Export format(s): plain text file

Publication: Tanaka, N., Matsui, T., Shimada, K., Yagihashi, T., and Taoda, H. (2005) Constructing Vegetation Databases Useful for Assessing Impact of Climate Changes in Japan. Journal of Agricultural Meteorology 60: 433-438.

Plot type(s): normal plots

Non-overlapping plots: 39,287

Total plot observations: 39,287

Countries: JP: $100.0 \%$

Forest: [NA] - Non-forest: [NA]

Guilds: all vascular plants: $100 \%$; bryophytes (terricolous or aquatic): $1 \%$; lichens (terricolous or aquatic): $1 \%$; non-terricolous taxa (epiphytic, saxicolous, lignicolous): $5 \%$

Environmental data: altitude: $90 \%$; slope aspect: $70 \%$; slope inclination: $70 \%$; microrelief: $40 \%$; surface cover other than plants (open soil, litter, bare rock etc.): $20 \%$

Performance measure(s): presence/absence only: $5 \%$; cover: $95 \%$

Geographic localisation: GPS coordinates (precision $25 \mathrm{~m}$ or less): $1 \%$; point coordinates less precise than GPS, up to $1 \mathrm{~km}$ : $4 \%$; small grid (not coarser than $10 \mathrm{~km}): 70 \%$; political units or only on a coarser scale $(>10 \mathrm{~km}): 25 \%$

Sampling periods: $[N A]$

Information as of 2012-07-12; further details and future updates available from http://www.givd.info/ID/AS-JP-001

Nobuyuki Tanaka (ntanaka@affrc.go.jp)

Department of Plant Ecology, Forestry and Forest Products Research Institute, Matsunosato 1, 305-8687 Tsukuba, JAPAN 